\title{
TRAVELTIME COMPUTATION FOR QSV WAVES IN TI MEDIA USING PHYSICS-INFORMED NEURAL NETWORKS
}

\author{
U.B. Waheed ${ }^{1}$, T. Alkhalifah ${ }^{2}$, B. Li $^{3}$, E. Haghighat ${ }^{4}$, A. Stovas ${ }^{3}$, J. Virieux ${ }^{5}$
}

${ }^{1}$ KFUPM $;{ }^{2}$ KAUST; ${ }^{3}$ NTNU; ${ }^{4}$ MIT; $;{ }^{5}$ Université Grenoble Alpes

\section{Summary}

Traveltimes corresponding to both compressional and shear waves are needed for many applications in seismology ranging from seismic imaging to earthquake localization. Since the behavior of shear waves in anisotropic media is considerably more complicated than the isotropic case, accurate traveltime computation for shear waves in anisotropic media remains a challenge. Ray tracing methods are often used to compute qSV wave traveltimes but they become unstable around triplication points and, therefore, we often use the weak anisotropy approximation. Here, we employ the emerging paradigm of physics-informed neural networks to solve transversely isotropic eikonal equation for the qSV wave that otherwise are not easily solvable using conventional finite difference methods. By minimizing a loss function formed by imposing the validity of eikonal equation, we train a neural network to produce traveltime solutions that are consistent with the underlying equation. Through tests on synthetic models, we show that the method is capable of producing accurate qSV wave traveltimes even at triplication points and works for arbitrary strength of medium anisotropy. 


\section{Traveltime computation for $\mathrm{qSV}$ waves in TI media using physics-informed neural networks}

\section{Introduction}

Traveltime computation of seismic waves is an essential ingredient for many applications in exploration and earthquake seismology. Traveltimes for compressional $(\mathrm{P})$ waves have been extensively used for imaging hydrocarbon reservoirs, statics and moveout correction, initial velocity model building for full waveform inversion, and microseismic source localization. Nevertheless, there are many good reasons to use shear (S) waves, for example, in imaging below salt or basalt domains where $\mathrm{S}$ waves have been shown to mitigate issues with $\mathrm{P}$ waves and in imaging when fluid content is involved (Garotta, 1999). Furthermore, $\mathrm{S}$ waves are necessary to ascertain lithological information, such as the Poisson's ratio. Moreover, since the sensitivity to azimuthal anisotropy is higher for $\mathrm{S}$ waves compared to $\mathrm{P}$ waves, the $S$ wave contribution may enhance the study of fracturing in reservoirs. Inverted $S$ wave images and velocity models tend to have higher resolution than $\mathrm{P}$ waves, considering the shorter wavelength associated with the slower $\mathrm{S}$ waves. Therefore, an accurate and efficient traveltime computation tool for $\mathrm{S}$ waves is necessary for the success of these applications.

Seismic wave propagation markedly differs in anisotropic media compared to the isotropic case. In contrast to having only the $\mathrm{P}$ and $\mathrm{S}$ waves in isotropic media, the presence of anisotropy results in three wave modes - one quasi-compressional ( $\mathrm{qP}$ ) wave and two quasi-shear $\left(\mathrm{qS}_{1}\right.$ and $\left.\mathrm{qS}_{2}\right)$ waves. We refer to these quasi-shear wave modes in transversely isotropic (TI) media as the horizontally polarized (qSH) and vertically polarized (qSV) shear waves. Most of the research efforts in solving the anisotropic eikonal equation have revolved around computing the $\mathrm{qP}$ wave traveltimes for TI media using the popular fast sweeping algorithm. Asymptotic approaches allow traveltime computation independent of the $\mathrm{P}$ or $\mathrm{S}$ wave traveltimes. While the computation of $\mathrm{qSH}$ wave traveltimes is similar to the $\mathrm{qP}$ case, solving qSV traveltimes should overcome the presence of triplications (Padhi and Willis, 2019; Huang et al., 2020). When performing ray tracing, one should avoid unexpected jumps between branches by constraining the slownesss vector sampling (Vavryčuk, 2001). However, computing the viscosity solution may automatically select the correct fastest branch.

Here, we propose an anisotropic eikonal solver to compute qSV wave traveltimes based on the emerging paradigm of physics-informed neural networks (PINNs). PINNs leverage the capabilities of neural networks as universal function approximators. However, contrary to conventional deep learning approaches, PINNs restrict the space of admissible solutions by imposing the validity of the partial differential equation (PDE) through the loss function. PINNs have already been successfully used to model seismic traveltime and wavefields solutions (Waheed et al., 2020; Song et al., 2020). Based on a loss function defined by the underlying anisotropic eikonal equation for qSV waves, we train a neural network to yield qSV wave traveltimes. Through tests on benchmark synthetic models, we show that the method is capable of providing accurate traveltime solutions without any limits on the strength of medium anisotropy. Moreover, it allows us to transfer information gained by solving one problem to the next by using the concept of transfer learning. This could be crucial in speeding up the training process for updated models and/or source locations.

\section{Theory}

In a 2D TI medium with vertical axis of symmetry (VTI), the qP and qSV waves are coupled, forming a quartic slowness surface, given as (Han et al., 2017):

$$
p_{z}^{4}+B p_{z}^{2}+C=0
$$

where

$$
\begin{aligned}
& B=\frac{1}{v_{p}^{2}}+\frac{1}{v_{s}^{2}}-2\left(1+\delta+(\varepsilon-\delta) \frac{v_{p}^{2}}{v_{s}^{2}}\right) p_{x}^{2} \\
& C=\left((1+2 \varepsilon) p_{x}^{2}-\frac{1}{v_{p}^{2}}\right)\left(p_{x}^{2}-\frac{1}{v_{s}^{2}}\right) .
\end{aligned}
$$


In the above equations, $p_{x}$ and $p_{z}$ represent the horizontal and vertical slowness components, respectively; $v_{p}$ and $v_{s}$ denote the $\mathrm{P}$ and $\mathrm{S}$ wave phase velocities along the vertical axis, respectively; $\varepsilon$ and $\delta$ are the Thomsen parameters. The two roots of equation (1) represent the $\mathrm{qP}$ and $\mathrm{qSV}$ wave slowness surfaces. Since qSV waves travel slower than qP waves, the larger root

$$
p_{z}^{2}-\frac{B+\sqrt{B^{2}-4 C}}{2}=0
$$

corresponds to the qSV slowness surface (the qP solution is the smaller other root not studied here). By replacing slowness components by

$$
p_{x}=\frac{\partial T}{\partial x}, \quad p_{z}=\frac{\partial T}{\partial z}
$$

one can compute the qSV wave traveltime $T(x, z)$ from a point-source to a point $(x, z)$ in the computational domain. For avoiding the point-source singularity, we factor the unknown traveltime $T$ into two multiplicative functions, such that

$$
p_{x}=T_{0} \frac{\partial \tau}{\partial x}+\tau \frac{\partial T_{0}}{\partial x}, \quad p_{z}=T_{0} \frac{\partial \tau}{\partial z}+\tau \frac{\partial T_{0}}{\partial z},
$$

where the known factor $T_{0}$ is computed analytically while the unknown factor $\tau$ is smooth around the source. We substitute $p_{x}$ and $p_{z}$ into equation (3) and solve for $\tau$. For a tilted transversely isotropic (TTI) medium, the slowness components, given in equation (5), are rotated by using a local rotation angle $\theta(x, z)$ between the local rotation symmetry axis and the vertical axis.

To solve equation (3) using a deep neural network (DNN), we consider a network with two neurons in the input layer for the spatial coordinates $(x, z)$, an output neuron for the estimated traveltime factor $\hat{\tau}(x, z)$, and a number of hidden layers. The partial derivatives of the output $(\hat{\tau})$ w.r.t the inputs $(x, z)$ can be computed using automatic differentiation. The loss function that we seek to minimize is:

$$
\mathfrak{J}=\frac{1}{N_{I}} \sum_{\left(x^{*}, z^{*}\right) \in I}\|\mathscr{L}\|^{2}+\frac{1}{N_{I}} \sum_{\left(x^{*}, z^{*}\right) \in I}\|\mathscr{H}(-\hat{\tau}) \mid \hat{\tau}\|^{2}+\left\|\hat{\tau}\left(x_{s}, z_{s}\right)-1\right\|^{2},
$$

where $\mathscr{L}$ represents the residual of the factored qSV eikonal equation, given by plugging $p_{x}$ and $p_{z}$ from equation (5) into (3). Therefore, the first term on the right hand side of equation (6) imposes the validity of the qSV eikonal equation on a given set of training points $\left(x^{*}, z^{*}\right) \in I$, where $N_{I}$ is the number of training samples. The second term forces the solution to be positive, where $\mathscr{H}()$ is the Heaviside function and it is used to penalize negative solutions. The last term enforces the boundary condition.

The set of network parameters $\theta^{*}$ that minimizes the loss function (6) on this set of training points, $\left(x^{*}, z^{*}\right) \in I$, is then identified by solving the optimization problem:

$$
\theta^{*}=\arg \min _{\theta} \mathfrak{J}\left(x^{*}, z^{*} ; \theta\right) .
$$

Once the DNN is trained, we evaluate the network on a set of regular grid-points in the computational domain to obtain the unknown traveltime field. The final qSV traveltime solution is obtained by multiplying the predicted traveltime with the known traveltime part, i.e.,

$$
\hat{T}(x, z)=T_{0}(x, z) \hat{\tau}(x, z) .
$$

\section{Results}

In this section, we test the proposed approach in computing traveltimes corresponding to the qSV wave. The DNN model is implemented using the SciANN (Haghighat and Juanes, 2021) package - a highlevel Tensorflow wrapper for scientific computations. For each of the examples presented below, we use a neural network having 20 hidden layers with 100 neurons in each layer and minimize the loss 
function using full-batch optimization. A locally adaptive inverse tangent activation function is used for all hidden layers except the final layer, where we use a linear activation function. Locally adaptive activation functions have been shown to achieve superior optimization performance and convergence speed over base methods (Jagtap et al., 2020). To benchmark, we compare the traveltime solution against the qSV wavefield solution obtained using the low-rank method (Fomel et al., 2013).

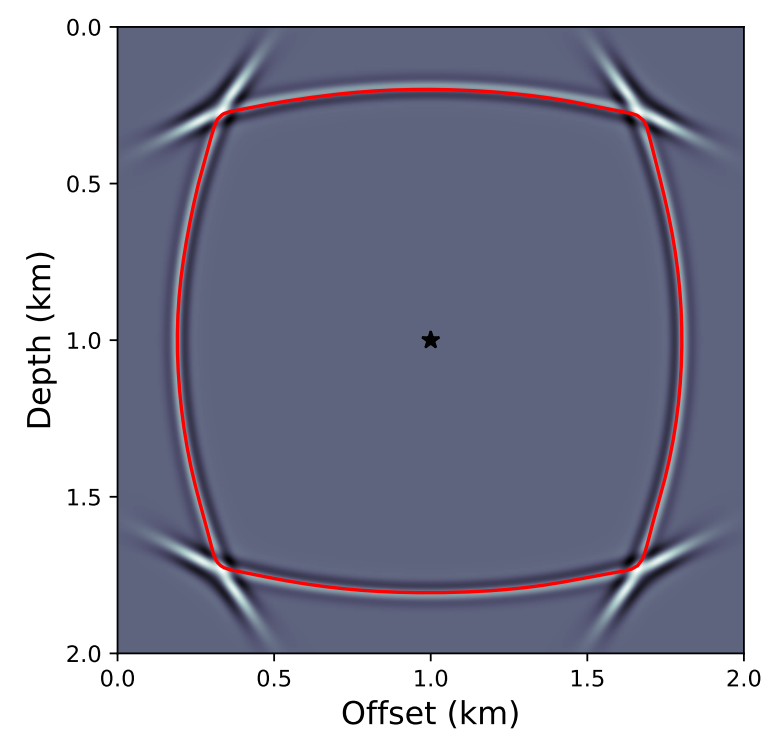

Figure 1 Traveltime contour (red) for the qSV wave at $0.5 \mathrm{~s}$ overlaid on top of the corresponding wavefield snapshots for the homogeneous VTI model. Black star indicates the source position.

We begin by considering a $2 \times 2 \mathrm{~km}^{2}$ homogeneous VTI model based on the Greenhorn shale sample with $v_{p}=3.094 \mathrm{~km} / \mathrm{s}, v_{s}=1.51 \mathrm{~km} / \mathrm{s}, \varepsilon=0.256$, and $\delta=-0.0505$ discretized on a $101 \times 101$ grid with a grid spacing of $20 \mathrm{~m}$ along both axes. For a point-source located at $(1 \mathrm{~km}, 1 \mathrm{~km})$, we train a DNN on $50 \%$ of the total grid points selected randomly. The optimization is performed first using Adam optimizer followed by an L-BFGS-B step, each run lasting 5,000 epochs. Figure 1 shows a traveltime contour overlaid on top of the qSV wavefield solution, both taken at $0.5 \mathrm{~s}$. By visual observation, we confirm that the traveltime solution matches well with the main branch of the wavefield. The accuracy of the solution is remarkably high even at the triplication point, which is often the source of complication for conventional algorithms.

Next, we show how transfer learning can be used to expedite training for modified model parameters. We consider a $2 \times 2 \mathrm{~km}^{2}$ model with linearly increasing velocity. The $\mathrm{P}$ wave velocity, $v_{p}$, goes from $2 \mathrm{~km} / \mathrm{s}$ at zero depth to $3 \mathrm{~km} / \mathrm{s}$ at a depth of $2 \mathrm{~km}$. The $\mathrm{S}$ wave velocity model, $v_{s}$, is obtained by dividing $v_{p}$ by a constant factor of 2 . The anisotropy parameters and tilt angle are taken as constants: $\varepsilon=0.4, \delta=0.2$, and $\theta=40^{\circ}$. The model is discretized on a $101 \times 101$ grid with a grid spacing of $20 \mathrm{~m}$ along both axes. Instead of training from scratch, we use pre-trained weights for the DNN obtained from training of the homogeneous VTI example. Starting with a pre-trained network allows us to use the L-BFGS-B method directly for faster convergence as opposed to starting with the Adam optimizer and then switching to L-BFGS-B as in the previous example. For a point-source located at $(1 \mathrm{~km}, 1 \mathrm{~km})$, Figure 2 shows a traveltime contour overlaid on top of the corresponding qSV wavefield solution taken at $0.6 \mathrm{~s}$. Again, we observe a good match between the traveltime and wavefield solutions. Moreover, the traveltime solution is obtained after 3000 L-BFGS-B epochs, saving considerable compute time compared to training from scratch.

\section{Conclusions}

We proposed an algorithm to compute qSV wave traveltimes based on the framework of physicsinformed neural networks. We factor the traveltime into two functions: one is obtained analytically while the other is obtained by leveraging the capabilities of neural networks as universal function ap- 


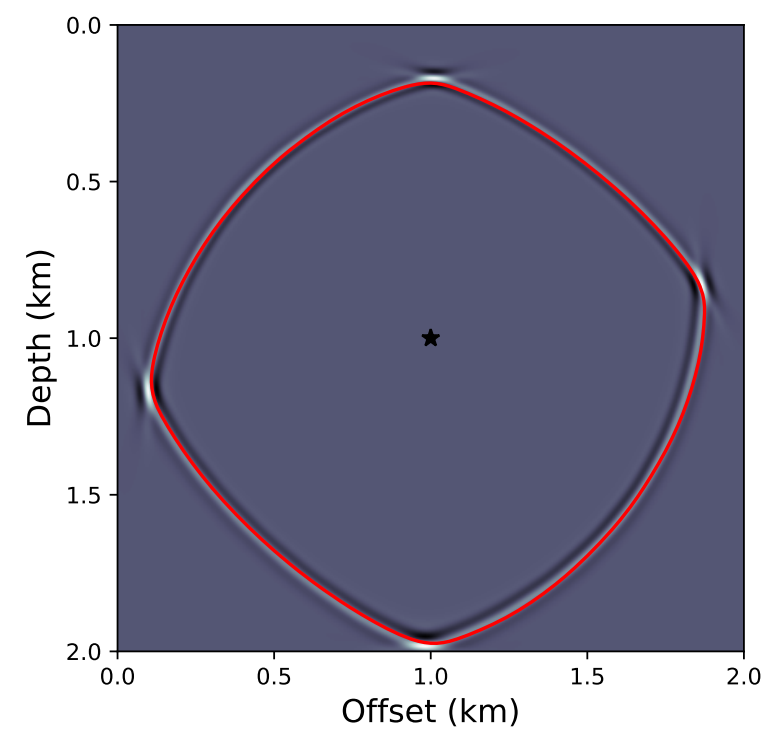

Figure 2 Traveltime contours (red) for the qSV wave at $0.6 s$ overlaid on top of the corresponding wavefield snapshots for the TTI model with linearly increasing velocity. Black star indicates the source position.

proximators to estimate the unknown factor. This is achieved with a feed-forward neural network using automatic differentiation. As opposed to conventional techniques, our method works for arbitrary strength of anisotropy. The presence of triplications leads to multi-valued traveltimes; however, the method provides us with the solution corresponding to the main branch of the qSV wave only. Additional research is needed to guide the neural network to output multi-valued solutions.

\section{References}

Fomel, S., Ying, L. and Song, X. [2013] Seismic wave extrapolation using lowrank symbol approximation. Geophysical Prospecting, 61(3), 526-536.

Garotta, R. [1999] Shear waves from acquisition to interpretation. Society of Exploration Geophysicists.

Haghighat, E. and Juanes, R. [2021] SciANN: A Keras/TensorFlow wrapper for scientific computations and physics-informed deep learning using artificial neural networks. Computer Methods in Applied Mechanics and Engineering, 373, 113552.

Han, S., Zhang, W. and Zhang, J. [2017] Calculating qP-wave traveltimes in 2-D TTI media by highorder fast sweeping methods with a numerical quartic equation solver. Geophysical Journal International, 210(3), 1560-1569.

Huang, G., Luo, S., Deng, J. and Vavryčuk, V. [2020] Traveltime Calculations for qP, qSV, and qSH Waves in Two-Dimensional Tilted Transversely Isotropic Media. Journal of Geophysical Research: Solid Earth, 125(8), e2019JB018868.

Jagtap, A.D., Kawaguchi, K. and Em Karniadakis, G. [2020] Locally adaptive activation functions with slope recovery for deep and physics-informed neural networks. Proceedings of the Royal Society A, 476(2239), 20200334.

Padhi, A. and Willis, M. [2019] Accurate quasi-SV traveltimes in 3D transversely isotropic media with vertical axis of symmetry using a high-order fast-sweeping-based eikonal solver. In: SEG Technical Program Expanded Abstracts 2019, Society of Exploration Geophysicists, 3879-3883.

Song, C., Alkhalifah, T. and Waheed, U. [2020] Solving the acoustic VTI wave equation using physicsinformed neural networks. arXiv preprint arXiv:2008.01865.

Vavryčuk, V. [2001] Ray tracing in anisotropic media with singularities. Geophysical Journal International, 145, 265-276.

Waheed, U., Haghighat, E., Alkhalifah, T., Song, C. and Hao, Q. [2020] Eikonal solution using physicsinformed neural networks. arXiv preprint arXiv:2007.08330. 